\title{
Multiparametric magnetic resonance tomography and MRI/TRUS-fusion-biopsy for index lesion detection: correlation with radical prostatectomy specimen
}

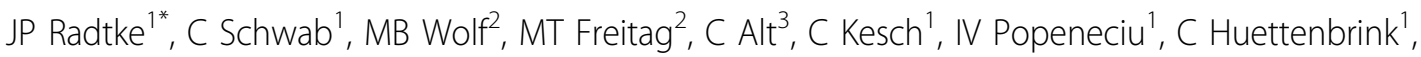 \\ C Bergstraesser-Gasch', T Klein', S Duensing ${ }^{1}$, S Roth ${ }^{4}$, HP Schlemmer ${ }^{2}, M_{\text {Roethke }}^{2}$, M Hohenfellner ${ }^{1}$, \\ B Hadaschik ${ }^{1}$ \\ From International Cancer Imaging Society Meeting and 15th Annual Teaching Course (ICIS 2015) \\ London, UK. 5-7 October 2015
}

\begin{abstract}
Aim
Multiparametric MRI (mpMRI) and MRI-targeted fusionbiopsy (TB) detect significant prostate cancer (PC) more accurately than conventional biopsies alone. The aim of this study was to evaluate the detection accuracy by mpMRI and TB on radical prostatectomy (RP) specimen.
\end{abstract}

\section{Methods}

We selected 120 consecutive patients who underwent transperineal fusion-biopsy before RP. All men received a saturation biopsy (SB) in addition to targeted biopsies of lesions with PIRADS $\geq 2$. On RP specimen, the index lesion was defined as highest Gleason score (GS) or highest tumour volume (TV). GS $=3+3$ and $\mathrm{TV} \geq 1.2 \mathrm{ml}$ or $\mathrm{GS}=3+4$ and $\mathrm{TV} \geq 0.7 \mathrm{ml}$ or $\mathrm{GS}>3+4$ were considered significant PC. We performed Spearmans correlation analysis between mpMRI and RP and Fisher`s test between mpMRI, TB and SB.

\section{Results}

Overall, 120 index lesions and 71 non-index lesions were detected. 107 index and 51 non-index lesions harbored significant PC. MpMRI detected 110/120(91.7\%) index lesions, while TB alone diagnosed only $96 / 120(80.0 \%)$ and SB alone 110/120(91.7\%). The combination of SB and TB detected 115/120(95.8\%) index foci. The combination of

\footnotetext{
* Correspondence: j.radtke@dkfz-heidelberg.de

${ }^{1}$ Department of Urology University Hospital Heidelberg, Heidelberg, Germany

Full list of author information is available at the end of the article
}

TB and SB outperformed TB alone ( $p=0.017)$ for detection of all significant PC. Additionally, TB performed significantly worse compared to SB alone for all significant tumour detection $(p=0.034)$. Spearmans correlation coefficient for index lesion concordance between mpMRI and $\mathrm{RP}$ was $0.865(p<0.001)$. TB provided greatest benefit in men undergoing repeat biopsy.

\section{Conclusions}

MpMRI detected 91.7\% index lesions compared to RP. However, TB alone missed $21.5 \%$ of all significant foci. Thus, the combination of both biopsy approaches should be incorporated in the biopsy workflow to predict PC most accurately.

\section{Authors' details \\ 'Department of Urology University Hospital Heidelberg, Heidelberg, Germany. ${ }^{2}$ Department of Radiology German Cancer Research Center Heidelberg, Heidelberg, Germany. ${ }^{3}$ Department of Diagnostic and Interventional Radiology University, Hospital Dusseldorf, Dusseldorf, Germany. ${ }^{4}$ Institute of Pathology University Hospital, Heidelberg, Germany.}

Published: 2 October 2015

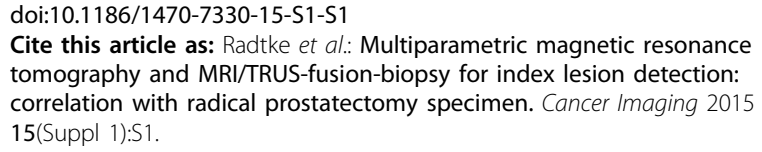

Cite this article as: Radtke et al:: Multiparametric magnetic resonance tomography and MRI/TRUS-fusion-biopsy for index lesion detection: correlation with radical prostatectomy specimen. Cancer Imaging 2015 15(Suppl 1):S1. 